\title{
PERCEPCIÓN DEL MÉTODO DEL CASO COMO HERRAMIENTA DE ENSEÑANZA EN UN CURSO DE EMPRENDIMIENTO
}

\author{
PERCEPÇÃO DO MÉTODO DE ESTUDO DE CASO COMO \\ FERRAMENTA DE ENSINO EM UM CURSO DE \\ EMPREENDEDORISMO
}

\section{PERCEPTION OF THE CASE METHOD AS A TEACHING TOOL IN AN ENTREPRENEURSHIP COURSE}

\author{
Franklin Duarte Cueva ${ }^{1}$
}

Artigo recebido em junho de 2021

Artigo aceito em agosto de 2021

\section{RESUMEN}

Este artículo se relaciona con el uso del método del caso como herramienta didáctica en el proceso de enseñanza, en un curso universitario, para lograr los objetivos de aprendizaje. Actualmente, la actividad emprendedora influye en la dinámica económica, social y cultural de los países, convirtiéndose en un eje clave para los gobiernos, quienes toman decisiones para promover el emprendimiento y la educación emprendedora. La investigación se centra en el curso emprendimiento, el cual se imparte en una universidad, a nivel de estudios de pre grado. El objetivo general de la investigación es conocer la percepción del estudiante universitario acerca del uso del método del caso como herramienta de enseñanza. Se trabajó con una muestra de ex estudiantes del curso, correspondiente a tres semestres académicos consecutivos. Como instrumento de medición se diseñó una guía de proposiciones con escala de Likert, la cual fue validada y se midió la fiabilidad del instrumento. Los resultados muestran que el método del caso es una herramienta didáctica valorada positivamente por los estudiantes.

Palabras clave: Educación emprendedora. Emprendimiento. Herramientas de enseñanza. Método del caso. Taxonomía revisada de Bloom.

\section{RESUMO}

Este artigo trata da utilização do método de estudo de caso como ferramenta didática no processo de ensino, em um curso universitário, para o alcance dos objetivos de aprendizagem. Atualmente, a atividade empreendedora influencia a dinâmica econômica, social e cultural dos países, tornando-se um eixo fundamental para os governos, que tomam decisões para promover o empreendedorismo e a educação empreendedora. A pesquisa tem como foco o curso de Empreendedorismo, que é ministrado em uma universidade, em nível de graduação. O objetivo geral da pesquisa é conhecer a percepção do estudante universitário sobre a utilização do método de estudo de caso como ferramenta de ensino. Trabalhamos com uma amostra de ex-alunos do curso, correspondendo a três semestres letivos consecutivos. Como instrumento de medida, foi elaborado um guia de propostas com escala Likert, o qual foi validado, e a confiabilidade do instrumento medida. Os resultados mostram que o método de estudo de caso é uma ferramenta didática valorizada positivamente pelos alunos.

\footnotetext{
${ }^{1}$ Profesor Asociado de la Pontificia Universidad Católica del Perú. E-mail: fduarte@pucp.edu.pe.
} 
Palavras-chave: Educação empreendedora. Empreendedorismo. Ferramentas de ensino. Método de caso. Taxonomia revisada de Bloom.

\begin{abstract}
This article is related to the use of the case method as a didactic tool in the teaching process, in a university course, to achieve the learning objectives. Currently, entrepreneurial activity influences the economic, social and cultural dynamics of countries, becoming a key axis for governments, who make decisions to promote entrepreneurship and entrepreneurial education. The research focuses on the entrepreneurship course, which is taught at a university, at the undergraduate level. The general objective of the research is to know the perception of the university student about the use of the case method as a teaching tool. We worked with a sample of former students of the course, corresponding to three consecutive academic semesters. As a measurement instrument, a proposal guide with a Likert scale was designed, which was validated and the reliability of the instrument was measured. The results show that the case method is a didactic tool valued positively by the students.
\end{abstract}

Keywords: Entrepreneurial education. Entrepreneurship. Teaching tools. Case method. Bloom's revised taxonomy.

\title{
1 INTRODUCCIÓN
}

Comienza la introducción por emprendimiento.

\subsection{Emprendimiento}

La descripción del emprendedor comienza con Schumpeter (1912) enfatizó el rol del emprendedor como causa esencial del desarrollo económico y describe como el emprendedor innovador desafía a las firmas establecidas, introduciendo nuevas invenciones que hacen que las tecnologías y productos actuales sean obsoletos.

De acuerdo con Drucker (1985) el emprendimiento no es mágico, no es misterioso y no tiene nada que ver con los genes. Es una disciplina, y como cualquier disciplina, se puede aprender. Shane y Venkataraman (2000) dicen que emprendimiento es el estudio de los tipos de oportunidades para crear algo nuevo a través de un proceso de descubrimiento, exploración y evaluación, y el individuo utiliza diferentes medios para llegar a su fin. Para, Reynolds et al. (2005) emprendimiento es cualquier intento de crear nuevos negocios o nuevas empresas, como por ejemplo, el autoempleo, la creación de una organización empresarial, o la expansión de un negocio existente; llevado a cabo por un individuo, un equipo de personas, o un negocio establecido.

Según SEBRAE (s/f) Emprendimiento es la capacidad de una persona para identificar problemas y oportunidades, desarrollar soluciones e invertir recursos en la creación de algo positivo para la sociedad.

A nivel específico se puede hablar del emprendedor, el cual es el protagonista de la actividad emprendedora.

Una persona creativa, marcada por la capacidad de establecer y alcanzar objetivos y que mantiene un alto nivel de consciencia del entorno en el que vive, usándolo para detectar oportunidades de negocio, utilizando la innovación y asumiendo riesgos calculados (FILION, 1999). 
Desde otra perspectiva Neck et al. (2020) sostiene que el emprendimiento es una manera de pensar, actuar y ser que combina la capacidad para encontrar o crear nuevas oportunidades, con el coraje para actuar sobre ellas. En general, el emprendimiento es una actividad humana desarrollada por los emprendedores para identificar oportunidades de negocio, con el fin de crear nuevas empresas generando valor y asumiendo riesgos, en la búsqueda por satisfacer necesidades actuales y futuras de la sociedad, usando una metodología interactiva, adaptativa y eminentemente práctica.

\subsection{Importancia del emprendimiento}

Uno de los reportes líderes sobre emprendimiento llamado Global Monitor Entrepreneurship (2019/2020) señala que el emprendimiento es un mecanismo excepcionalmente poderoso para el desarrollo económico y social que genera ingresos y puestos de trabajo al tiempo que fortalece y enriquece a las personas y las comunidades. En el contexto global, muchos países consideran al emprendimiento como un pilar esencial para el desarrollo nacional. La relevancia del emprendimiento también se refleja en la apreciación de Thurik y Wennekers (1999) cuando refieren que en el mundo actual las pequeñas empresas, y particularmente las nuevas, son vistas más que nunca como un vehículo para el emprendimiento, contribuyendo no solo al empleo y la estabilidad social y política sino también al poder innovador y competitivo de un país. Es demostrable la contribución de las pequeñas empresas en la economía de países desarrollados como por ejemplo Alemania y Corea del Sur.

La gran esperanza de nuestra sociedad son las nuevas empresas capaces de crecer con dinamismo. Por suerte estas empresas existen y la renovación del tejido económico que realizan permite crear nuevos puestos de trabajo. Ellas aportan además, innovación estímulo y desafío professional (NUENO, 2009).

Creemos que hay una diversidad de estudios que convergen en la idea que el emprendimiento es una actividad vital en la sociedad porque es transversal al entorno económico, social, político, legal, demográfico, tecnológico, ambiental y sanitario. En este contexto, Filion et al. (2011) señala que el desarrollo económico contemporáneo depende en gran medida del desenvolvimiento de las pequeñas y medianas empresas, tal como demuestran las tasas de crecimiento de éstas en países europeos y asiáticos, cuyos gobiernos se esfuerzan por implementar políticas que favorezcan a este tipo de organizaciones a sabiendas de que es el mejor modo de impulsar la buena marcha de la economía nacional.

Las oportunidades no deberían estar limitadas a las empresas de gran tamaño. En este sentido, una mayor inserción internacional de las pequeñas y medianas empresas, además de contribuir a la generación de empleo y al incremento de los ingresos, brinda la posibilidad de alcanzar mayores niveles de productividad e innovación tecnológica. Este puede ser un camino para que algunas Pymes superen deficiencias y limitaciones. Para ello es necesario impulsar políticas públicas y acciones con los agentes privados en dos frentes a fin de romper el círculo vicioso que condiciona y limita el desempeño de las Pymes CEPAL (2013). 
Si hablamos de una mayor internacionalización de las Pymes, es prioritario considerar como mecanismo impulsor, la capacitación constante de emprendedores, empresarios y estudiantes universitarios, principalmente, porque éstos últimos, constituirán la fuerza laboral y una fuente de innovación en el corto y mediano plazo.

En el contexto latinoamericano, de acuerdo con Brenes y Haar (2017), en la primera década del siglo XXI, durante los años del boom de 2005-2009 y las etapas de "gran recesión" y "recuperación anémica" que siguieron, el emprendimiento hizo frente y se centró tanto en los emprendedores por oportunidad como en los emprendedores por necesidad quienes aprovecharon el espíritu empresarial para crear algo propio, independientemente de la orientación política -socialista, capitalista o mixta- muchos países han estado promoviendo el emprendimiento mediante diversos mecanismos de política y programas.

Posteriormente, manifiestan que el emprendimiento es crucial para el desarrollo de las economías emergentes porque es un motor que impulsa la inversión, el empleo y la innovación. Por lo tanto, es importante determinar qué factores promueven el surgimiento de empresarios. Por ejemplo, un sector financiero bien desarrollado, un sistema de disputas comerciales transparente, un mercado laboral calificado y la protección de la inversión, son factores positivos a nivel social. A nivel individual, los factores como las actitudes individuales hacia el riesgo y la exposición personal a una cultura empresarial, se vuelven cada vez más importantes.

En América Latina, tenemos millones de emprendedores por necesidad, los cuales forman parte de la denominada economía informal, siendo dichas personas las generadoras de sus propias fuentes de trabajo y son quienes asumen los mayores riesgos. Los emprendedores por necesidad han aumentado debido a los efectos negativos de la pandemia sobre la mano de obra como producto del cierre y reducción de micro, pequeños, medianos y grandes negocios formales. Creemos que es prioritario formalizar y educar a los emprendedores informales para mejorar su competitividad, de tal forma, que ellos puedan contribuir con la recaudación tributaria de manera justa, se conviertan en agentes generadores de trabajo formal y desincentiven las prácticas de competencia desleal en los mercados.

\subsection{Emprendimiento en el Perú}

En el Perú, debido a la desigual distribución del ingreso nacional, la migración del campo a la ciudad, el ingreso de migrantes internacionales, el crecimiento del número de familias, la falta de oportunidades laborales, las dificultades para acceder a financiamiento y el dispar acceso a la prestación de servicios educativos de calidad, entre otros aspectos; se genera una mayor proporción de emprendimientos por necesidad, más aún en épocas de crisis económicas o de pandemias como la que se vive actualmente.

De acuerdo con Serida et al. (2018), el reporte Global Entrepreneurship Monitor Perú 2017-2018, señala que el 64.7\% de peruanos consideran que en el Perú el emprendimiento es una buena elección de carrera. En este contexto, de los emprendedores motivados por oportunidad, el 59.3\% cuenta con estudios superiores, mientras que en aquellos motivados por necesidad, sólo el $35.3 \%$ ha alcanzado ese mismo nivel de estudios. Es dentro del segmento de emprendedores con estudios superiores donde existe mayor potencial para el desarrollo de iniciativas con alto grado de innovación, por lo que este grupo resulta crucial para el desarrollo de programas y políticas de gobierno. Sin embargo, todos los grupos de emprendedores necesitan capacitarse constantemente para que adquieran mayores competencias y sus emprendimientos sean sostenibles. 
El mencionado estudio, considerando la evolución de la valoración de los expertos sobre las condiciones marco para el emprendimiento en el Perú 2007-2017 señala que una condición fundamental para el emprendimiento es la educación emprendedora a nivel técnico y universitario. En este sentido, creemos que los estudiantes universitarios que son emprendedores potenciales y varios de ellos son emprendedores nacientes, requieren estudiar cursos de emprendimiento para motivarlos y fortalecer su desempeño en la actividad emprendedora.

El estudio referido, proporciona estadísticas que indican que $30 \%$ de emprendedores en etapa temprana cuentan con educación superior universitaria, $24 \%$ de emprendedores en etapa temprana cuentan con educación superior técnica, 33\% de emprendedores en etapa temprana motivados por oportunidad cuentan con educación superior universitaria y el $26 \%$ de emprendedores en etapa temprana motivados por oportunidad cuentan con educación superior técnica. La actividad emprendedora en etapa temprana corresponde a una parte del proceso emprendedor que incluye a los emprendedores nacientes (negocio en marcha con hasta tres meses de operación) y a los emprendedores nuevos (negocio en marcha con menos de 3.5 años en el mercado).

Específicamente, no existe una carrera universitaria o técnica como tal en nuestro país, seguramente, en el futuro cercano podría hacerse realidad. Por otro lado, analizando la variable auto percepciones sobre emprendimiento, Perú presenta una alta tasa de temor al fracaso con un puntaje de 30.2 obteniendo el puesto 38 entre 49 países que conformaron la muestra.

En los reportes del Global Entrepreneurship Monitor (GEM) en que participó Perú, se mostró como uno de los países con la mayor tasa de nuevos emprendimientos relacionados a personas con edades entre 18 y 64 años, a nivel global, y es líder en América Latina. En contraposición, la tasa de mortalidad de los emprendimientos en Perú es elevada, aproximadamente el 50\% de nuevos negocios son descontinuados al finalizar el primer año.

Esta situación se origina por factores de mercado, la falta de planeación, la baja capacidad para lanzar emprendimientos que creen valor $\mathrm{y}$, la escasa capacitación en la educación secundaria y universitaria. En este contexto, Un segmento vital para potenciar el emprendimiento son los jóvenes universitarios y egresados quienes disponen de conocimientos que pueden facilitar e impulsar el emprendimiento. Estudiar un curso de emprendimiento que se imparte en carreras relacionadas con la actividad empresarial en la universidad, es un primer paso para los estudiantes que desean ingresar al mundo emprendedor.

\subsection{Justificación, problema, objetivos, límites y resumen del método}

La investigación se justifica porque el emprendimiento es considerado un factor fundamental en el crecimiento de la economía y el desarrollo social, en este contexto, resulta clave enseñar y sensibilizar a los estudiantes universitarios acerca de la importancia de la actividad emprendedora en la sociedad como un todo.

El problema de investigación fue planteado en la siguiente pregunta central: ¿Cómo percibe el estudiante universitario el uso del método del caso en la enseñanza de un curso de emprendimiento? Finalmente, el objetivo general de la investigación es conocer la percepción del estudiante universitario de una carrera relacionada con la actividad empresarial, acerca del uso del método del caso como herramienta de enseñanza, en un curso de emprendimiento. El estudio está limitado a una facultad relacionada con los negocios y a una universidad dentro 
del sistema universitario peruano en la cual se imparte el curso emprendimiento que corresponde al octavo semestre de estudios.

Se utilizó como instrumento una guía de proposiciones compuesta por once ítems que fueron evaluados por una muestra de ocho expertos y cuya fiabilidad fue determinada mediante el coeficiente alfa de Cronbach. El instrumento se aplicó a una muestra por conveniencia que estuvo integrada por ex estudiantes de tres semestres consecutivos, veinte por semestre, siendo veintiocho el promedio de estudiantes por semestre en el curso, en los últimos dos años.

\section{MARCO TEÓRICO}

Comienza el marco teórico por educación emprendedora.

\subsection{Educación emprendedora}

De acuerdo con QAA (2018), la educación para el emprendimiento tiene como objetivo construir sobre las competencias emprendedoras de los estudiantes que sean capaces de identificar oportunidades y desarrollar emprendimientos, convirtiéndose en auto empleados, estableciendo nuevos negocios o desarrollando y creciendo como parte de un riesgo empresarial. Para Hardie et al. (2020) los maestros necesitan oportunidades para construir confianza, conocimiento y capacidad con el fin de desarrollar experiencias de aprendizaje efectivas en la educación empresarial que sean relevantes para los estudiantes de hoy y los preparen para los desafíos de la vida futura. Neck y Greene (2011) afirman que acercarse al emprendimiento como método significa enseñar una manera de pensar y actuar basada en un conjunto de supuestos utilizando un portafolio de prácticas para fomentar la creación. El método obliga a los estudiantes a ir más allá de la comprensión, conocimiento y conversación. Requiere usar, aplicar y actuar. El método requiere práctica continua.

En opinión de Boldureanu et al. (2020) en las instituciones de educación superior, el aprendizaje del emprendimiento basado en modelos de rol puede promover la educación para el desarrollo sostenible. Varias perspectivas teóricas, como la teoría del capital humano, la autoeficacia empresarial y la teoría de la autodeterminación, sostienen que la educación emprendedora se correlaciona positivamente con las intenciones empresariales de los estudiantes, ya que proporciona conocimientos y habilidades adecuados y los motiva a desarrollar su carrera emprendedora. Neck et al. (2020) corrobora que el emprendimiento es un método y no un proceso. En este contexto, refiere que las razones que explicarían las altas tasas de fracaso de los pequeños nuevos negocios son la falta de educación emprendedora, la falta de desarrollo de la capacidad para superar el desorden, aceptar la ambigüedad y actuar incluso cuando no se está realmente seguro de qué hacer. Esta situación explicaría, con buena lógica, la creciente tendencia a enseñar emprendimiento en el ámbito educativo en diferentes países.

La inclusión de la iniciativa emprendedora en todas las etapas de la educación ha venido siendo objeto de debate en el ámbito internacional desde hace muchos años. La OCDE, y especialmente la Unión Europea, recomiendan desde hace tiempo a sus Estados miembro y a otras partes implicadas que lleven a cabo acciones 
decididas en esta materia (DIRECCIÓN GENERAL DE POLÍTICA DE LA PYME, 2010).

Por otro lado, el emprendimiento se viene constituyendo en una actividad que promueve la mejora de las condiciones de vida en la sociedad. De modo amplio, la enseñanza del emprendimiento a nivel mundial, la llevan a cabo universidades, institutos y organismos de promoción de la actividad emprendedora, principalmente de manejo estatal como por ejemplo el Servicio Brasilero de Apoyo a la Micro y Pequeña Empresa (SEBRAE), la Corporación de Fomento (CORFO) de Chile, el IPYME en España y la Administración de Pequeños Negocios de los Estados Unidos (SBA), entre otros.

Pensamos que, a nivel universitario, la enseñanza del emprendimiento demanda el uso de herramientas didácticas orientadas al logro de aprendizajes significativos, basadas en el desarrollo permanente de actividades prácticas relacionadas con el quehacer emprendedor, según el campo en que se pretenda emprender, como el emprendimiento lucrativo, emprendimiento social, emprendimiento cultural o el emprendimiento corporativo conocido como intraemprendimiento.

La Comisión Europea (2016) indica que el espíritu empresarial como competencia se aplica a todas las esferas de la vida. Permite a los ciudadanos nutrir su desarrollo personal, para contribuir activamente al desarrollo social, para ingresar al mercado laboral como empleado o auto empleado, y para iniciar o expandir emprendimientos que puedan tener un motivo cultural, social o comercial. En opinión de Paños (2017), el emprendimiento no solo se refiere a la creación de nuevas empresas y/o negocios. Actualmente, es entendido como una competencia que engloba un conjunto de habilidades y destrezas como son la disciplina, la creatividad, el liderazgo, el trabajo en equipo, la innovación, la toma de decisiones, entre otros, características con demanda en el ámbito personal, social y laboral.

\subsection{Herramientas didácticas}

Según el INACAP (2017), las herramientas didácticas o estrategias de enseñanza "son procedimientos organizados que tienen una clara formalización/definición de sus etapas y se orientan al logro de los aprendizajes esperados".

Es el conjunto de procedimientos, apoyados en técnicas de enseñanza que tienen por objeto llevar a buen término la acción didáctica, es decir, alcanzar los objetivos de aprendizaje (INSTITUTO TECNOLÓGICO DE MONTERREY, s/f).

En las universidades existe una amplia diversidad de herramientas de enseñanza. En este sentido, la naturaleza de la carrera académica, el perfil de los estudiantes y los recursos disponibles son algunos de los factores que influyen en el uso de determinados herramientas pedagógicas, siendo el objetivo que el estudiante aprenda o logre un aprendizaje.

En lo que hay consenso es en que el aprendizaje es el proceso o conjunto de procesos a través del cual o de los cuales, se adquieren o se modifican ideas, habilidades, destrezas, conductas o valores como resultado o con el concurso del estudio, la experiencia, la instrucción, el razonamiento o la observación. A esto habría que añadir unas características que tiene exclusivamente el aprendizaje: 
permite atribuir significado al conocimiento y permite atribuir valor al conocimiento (ZAPATA-ROS, 2015).

A continuación se presenta (Cuadro 1) una comparación general de las herramientas de enseñanza que pueden usarse en facultades relacionadas con el campo empresarial.

Cuadro 1 - Esquema comparativo de diversas herramientas de enseñanza

\begin{tabular}{|c|c|c|c|c|c|}
\hline $\begin{array}{c}\text { Criterios / } \\
\text { Herramienta }\end{array}$ & $\begin{array}{c}\text { Situación } \\
\text { problemática }\end{array}$ & $\begin{array}{l}\text { Modalidad } \\
\text { de trabajo }\end{array}$ & Complejidad & $\begin{array}{l}\text { Desarrollo de } \\
\text { competencias }\end{array}$ & $\begin{array}{l}\text { Rol del } \\
\text { docente }\end{array}$ \\
\hline $\begin{array}{l}\text { Aprendizaje } \\
\text { Basado en } \\
\text { Problemas }\end{array}$ & Ficticia & $\begin{array}{c}\text { Trabajo } \\
\text { individual y } \\
\text { en equipo }\end{array}$ & Baja/Media & Medio/Alto & $\begin{array}{l}\text { Docente } \\
\text { tutor }\end{array}$ \\
\hline $\begin{array}{l}\text { Aprendizaje } \\
\text { Basado en } \\
\text { Investigación }\end{array}$ & Ficticia o real & $\begin{array}{c}\text { Trabajo } \\
\text { individual y } \\
\text { en equipo }\end{array}$ & Media/Alta & Medio/Alto & $\begin{array}{l}\text { Docente } \\
\text { tutor }\end{array}$ \\
\hline $\begin{array}{l}\text { Aprendizaje } \\
\text { Basado en } \\
\text { Proyectos }\end{array}$ & $\begin{array}{l}\text { Basada en la } \\
\text { realidad }\end{array}$ & $\begin{array}{c}\text { Trabajo } \\
\text { individual y } \\
\text { en equipo }\end{array}$ & Media/Alta & Medio/Alto & $\begin{array}{l}\text { Docente } \\
\text { facilitador }\end{array}$ \\
\hline $\begin{array}{l}\text { Control de } \\
\text { Lectura }\end{array}$ & Ficticia o real & $\begin{array}{c}\text { Trabajo } \\
\text { individual }\end{array}$ & Baja/Media & Bajo & $\begin{array}{c}\text { Docente } \\
\text { protagonista }\end{array}$ \\
\hline Rompecabezas & Ficticia o real & $\begin{array}{c}\text { Trabajo en } \\
\text { equipo }\end{array}$ & Media & Medio & $\begin{array}{l}\text { Docente } \\
\text { facilitador }\end{array}$ \\
\hline Simulaciones & Ficticia & $\begin{array}{l}\text { Trabajo en } \\
\text { equipo }\end{array}$ & Alta & Alto & $\begin{array}{c}\text { Alumno } \\
\text { protagonista }\end{array}$ \\
\hline $\begin{array}{l}\text { Método del } \\
\text { Caso }\end{array}$ & $\begin{array}{l}\text { Basada en la } \\
\text { realidad }\end{array}$ & $\begin{array}{c}\text { Trabajo } \\
\text { individual y } \\
\text { en equipo }\end{array}$ & Media/Alta & Alto & $\begin{array}{c}\text { Docente } \\
\text { facilitador y } \\
\text { motivador }\end{array}$ \\
\hline Clase Magistral & Ficticia o real & $\begin{array}{l}\text { Trabajo } \\
\text { individual }\end{array}$ & Baja & Bajo & $\begin{array}{c}\text { Docente } \\
\text { protagonista }\end{array}$ \\
\hline Role Playing & Ficticia o real & $\begin{array}{c}\text { Trabajo } \\
\text { individual y } \\
\text { en equipo }\end{array}$ & Baja/Media & Medio & $\begin{array}{c}\text { Alumno } \\
\text { protagonista }\end{array}$ \\
\hline $\begin{array}{c}\text { Trabajo } \\
\text { Colaborativo }\end{array}$ & Ficticia o real & $\begin{array}{c}\text { Trabajo en } \\
\text { equipo }\end{array}$ & Baja/Media & Medio & $\begin{array}{l}\text { Docente } \\
\text { facilitador }\end{array}$ \\
\hline
\end{tabular}

Fuente: Adaptado de INACAP (2017) y aporte del autor

La presente investigación se orienta a explorar la percepción que tienen los estudiantes de una carrera relacionada con la actividad empresarial, acerca del uso del método del caso como herramienta de enseñanza, en un curso de emprendimiento que se imparte en la Facultad "N" de la universidad "A" (por cuestiones de confidencialidad no se identifican). En el curso de la facultad referida, se utiliza como herramientas didácticas el control de lectura, el método de casos, el aprendizaje basado en proyectos y la clase magistral. Respecto al método

$$
\text { Refas - ISSN 2359-182X v.7, n.6 }
$$


de casos, el profesor prepara cada caso vinculado a un emprendedor, incluyendo información cualitativa y cuantitativa del negocio y la economía.

\subsection{Taxonomía de Bloom y la taxonomía de Bloom revisada}

Según la Real Academia Española de la Lengua (RAE), taxonomía es la ciencia que trata de los principios, métodos y fines de la clasificación. Ampliando, podríamos decir que la taxonomía ayuda a sistematizar información y crear una clasificación categorizada para posibilitar el análisis y comprensión de una determinada materia. En el ámbito educativo, de acuerdo con Bloom (1956), la idea para un sistema de clasificación se formó en una reunión informal de los revisores en 1948 en la Convención de Asociación Americana de Psicología.

El trabajo de Bloom permitió representar el proceso de aprendizaje en sus diferentes niveles, a partir de ello, se facilitó la comprensión y elaboración de los objetivos de aprendizaje proceso de enseñanza. En opinión de Churches (2009) referencia que Bloom, un psicólogo educativo de la universidad de Chicago, propuso que el proceso de aprendizaje comprendía tres dominios psicológicos: el cognitivo relacionado con procesar información, conocimiento y habilidades mentales; el afectivo vinculado a actitudes y sentimientos, y el psicomotor que abarcaba habilidades manipulativas, manuales o físicas. Isaacs (1996) señala que para Bloom y sus colaboradores, los niveles jerárquicos del dominio cognitivo se explicaban de la siguiente manera:

a) Conocimiento: Recuerda ítems específicos;

b) Comprensión; Recuerda, pero puede hacer algo más (parafrasear, definir, discutir hasta cierto punto;

c) Aplicación: Todo lo anterior, pero puede tomar información de carácter abstracto y usarla en situaciones concretas;

d) Análisis: Desagrega una comunicación en sus partes constituyentes, revelando las relaciones entre ellas;

e) Síntesis: Une muchas partes o elementos desorganizados para formar un todo;

f) Evaluación: Emite juicios sobre el valor de materiales o métodos.

La jerarquía que estableció Bloom, es lo que más se cuestiona en la actualidad porque se ha comprobado que el aprendizaje puede iniciarse en diferentes niveles, sin seguir un ordenamiento específico y algunos se producen de manera simultánea. En este contexto, Krathwohl (2002) propuso la taxonomía revisada de las dimensiones de Bloom y en vez de usar sustantivos, pasó a usar verbos como puede verse en el gráfico siguiente.

Por ejemplo, la dimensión del conocimiento o dominio cognitivo, quedó explicada de la siguiente forma:

a) Recordar: Reconocer listar, describir, identificar, recuperar, denominar, localizar, encontrar;

b) Comprender: Interpretar, resumir, inferir, parafrasear, clasificar, comparar, explicar, ejemplificar;

c) Aplicar: Implementar, desempeñar, usar, ejecutar; 
d) Analizar: Comparar, organizar, deconstruir (deshacer analíticamente algo para darle una nueva estructura), atribuir, delinear, encontrar, estructurar, integrar;

e) Evaluar: Revisar, formular hipótesis, criticar, experimentar, juzgar, probar, detectar, monitorear;

f) Crear: Diseñar, construir, planear, producir, idear, trazar, elaborar (Cuadro 2).

Cuadro 2 - Clasificación del dominio cognitivo, Bloom versus Anderson/Krathwohl

\begin{tabular}{|c|c|}
\hline Propuesta de Bloom & $\begin{array}{c}\text { Propuesta de Anderson y Krathwohl } \\
\text { (Taxonomía revisada de Bloom) }\end{array}$ \\
\hline Evaluación & Crear \\
\hline Síntesis & Evaluar \\
\hline Análisis & Analizar \\
\hline Aplicación & Aplicar \\
\hline Comprensión & Comprender \\
\hline Conocimiento & Recordar \\
\hline
\end{tabular}

Fuente: adaptado de Bloom (1956) y Anderson/Krathwohl (2001)

Para efecto de la presente investigación, se utilizó la Taxonomía revisada de Bloom propuesta por Anderson y Krathwohl (2001) como marco conceptual para elaborar los ítems de la guía de proposiciones, la cual se sometió a un proceso de validación por ocho expertos. Luego, se aplicaron dichos ítems a ex alumnos del curso Emprendimiento de los tres últimos semestres académicos.

\subsection{El método del caso}

El método del caso tipificado por la representación de una situación de la realidad como cimiento para discutir y aprender, ha sido utilizado desde la antigüedad. Por ejemplo, la casuística, propia de la filosofía escolástica medieval, es la aplicación del caso para resolver problemas morales. Durante el renacimiento, especialmente en la enseñanza de la medicina, se utilizó la disección de cadáveres con el fin de no sólo estudiar los textos de Hipócrates y Galeno sino que los estudiantes se cuestionaran sobre la práctica.

De acuerdo con Harvard Law School (s/f), el método del caso en la educación jurídica fue inventado por Christopher Columbus Langdell, Decano de la Facultad de Derecho de Harvard de 1870 a 1895. Langdell concibió una forma de sistematizar y simplificar la educación jurídica centrándose en la jurisprudencia anterior que fomentaba principios o doctrinas. Con ese fin, Langdell escribió el primer libro de casos, titulado Una selección de casos sobre la ley de contratos, una colección de casos resueltos que iluminaría el estado actual de la ley de contratos. Los estudiantes leyeron los casos y vinieron preparados para analizarlos durante las sesiones de preguntas y respuestas socráticas en clase. Posteriormente, el método de casos fue usado en la Escuela de Medicina (HMS) en 1900 y después en la Escuela de Negocios (HBS) en 1908.

Según Estrada y Alfaro (2013) El método de casos tiene una correlación técnica y una metodológica a la vez, y se aplica con éxito en muchas disciplinas universitarias y en diversas 
actividades académicas. La enseñanza de este método siempre tiene diferencias y diversos puntos de vista y requiere cumplir con algunas condiciones relacionadas con la forma y el estilo necesarios para su eficaz desarrollo en el aula. El método es adecuado para la enseñanza universitaria porque no está basado sólo en la intervención o conocimientos del docente sino fundamentalmente en la participación activa del estudiante, quien identifica, analiza, sistematiza, evalúa, propone y decide cómo resolver las situaciones o problemas planteados en el caso.

Para Wasserman (1994), una característica obvia del método de casos es el uso de una herramienta educativa llamada caso. Los casos son instrumentos educativos complejos que toman la forma de narrativas. Un caso incluye información y datos: psicológicos, sociológicos, científicos, antropológicos, históricos y de observación, además de material técnico. Aunque los casos se centran en áreas temáticas específicas, por ejemplo, historia, pediatría, gobierno, derecho, negocios, educación, psicología, desarrollo, infantil, enfermería, etc., son por naturaleza interdisciplinarios. Por lo general, las narrativas se basan en problemas de la vida real.

Ogliastri (2009), explica que trabajar con casos en el aula, es una alternativa de enseñanza que involucra el auto-aprendizaje y el desarrollo de competencias del estudiante. El caso desencadena un proceso de pensamiento, de análisis, de solución de problemas o inclusive de evaluación y juicio. Bajo este supuesto, el proceso ayuda a formar estudiantes con la capacidad de enfrentar situaciones nuevas con criterios propios, pues la responsabilidad del profesor es facilitar que los estudiantes aprendan a pensar de manera independiente.

Rosker (2006), afirma que el caso se refiere a la descripción de una situación real que incluye un problema, una oportunidad, un desafío o la toma de una decisión de una persona dentro de una realidad organizacional y social. En definitiva, consiste en representar una determinada situación conflictiva y capacitar a los alumnos para que analicen la toma de decisiones resolutivas.

Camacho (2011) expresa que los métodos de casos especializados en la enseñanza o instrucción describen una situación real de una organización empresarial, institución o personas, en un contexto y en un período dado. Efectivamente, los casos se enmarcan en situaciones que han ocurrido o están ocurriendo en una determinada zona y en un momento del tiempo específico.

Para Boehrer and Linsky (2002), los propósitos de un caso en clases son adquirir un pensamiento crítico, proveer al estudiante de responsabilidades para su aprendizaje, transferir información, conceptos y técnicas, desarrollar comandos de todo el material, enlazar aprendizaje afectivo y cognitivo, desarrollar habilidades de colaboración y enseñar a cuestionarse bajo un esquema de aprendizaje auto adquirido.

Circunscribiéndonos al concepto de casos, aplicados a la actividad emprendedora, un caso refleja las experiencias que viven los emprendedores en diferentes etapas del ciclo de vida de su negocio, abarcando las etapas de ideación, lanzamiento, operaciones iniciales y desarrollo posterior; lo cual es influido por las acciones de los grupos de interés, cuya importancia varía de acuerdo con la naturaleza de la actividad económica que desarrolle la empresa.

Evidentemente, los casos acerca de emprendedores también revelan aspectos de la personalidad del emprendedor. Lo cual se refleja en su estilo de toma de decisiones y la manera en que interactúa con sus colaboradores y entes externos. Los casos vinculados a emprendimientos despiertan la curiosidad y el interés de los estudiantes porque les posibilita conectarse con el mundo real y pueden relacionar la teoría con la praxis, con lo cual puede esperarse la generación de aprendizajes significativos. En este contexto, resultaría interesante que el profesor trate de escribir sus propios casos. Se puede empezar con elaborar casos cortos de dos o tres páginas. En este caso, el docente elegirá un emprendedor y lo convencerá para 
hacerle una entrevista sobre la base de una guía de preguntas abiertas elaboradas previamente de acuerdo con los objetivos de aprendizaje.

Cuando un docente presenta un caso que el mismo planeó, formuló los objetivos, diseñó la guía de la entrevista, entrevistó al emprendedor, analizó e interpretó la información y escribió. Resulta algo extraordinario, porque el nivel de motivación, conocimiento, convicción y creación es mayor que cuando usamos casos escritos por otros autores, lo cual no significa que no se usen, hay casos interesantes que necesitamos trabajar en clase. Todo depende de la carga laboral del profesor, de las facilidades que otorguen las facultades y de la experiencia en el manejo de casos. Evidentemente, hay otras metodologías que también se usan en la enseñanza de un curso de emprendimiento las cuales complementan y apoyan el aprendizaje.

\section{MÉTODO}

Comienza el método por el tipo de investigación.

\subsection{Tipo de investigación}

La investigación es de tipo descriptivo y longitudinal. Hernández et al. (2010) refiere que los estudios descriptivos se orientan a especificar propiedades, características y perfiles de personas, grupos, comunidades, procesos o cualquier otro fenómeno que sea analizado; y es de tipo longitudinal porque el instrumento para la recolección de datos se aplicó en tres semestres académicos consecutivos.

\subsection{Población y muestra}

Para la presente investigación, el universo de estudio son los estudiantes de universidades privadas de la zona de Lima Metropolitana que han estudiado un curso de emprendimiento, en facultades relacionadas con la actividad empresarial. La muestra seleccionada está representada por ex estudiantes de tres semestres consecutivos del curso Emprendimiento de la facultad " $\mathrm{N}$ " vinculada con los negocios, quienes estudiaron en la Universidad "A". Las respuestas de cada participante son totalmente anónimas. Asimismo, por cuestiones de confidencialidad no se menciona el nombre de la universidad ni de la facultad. La muestra fue seleccionada por conveniencia, en concordancia con los siguientes criterios:

a) Los estudiantes cursaron la materia Emprendimiento en la Facultad " $N$ " de la universidad "A";

b) La universidad cuenta con licencia de funcionamiento institucional de la SUNEDU (Superintendencia Nacional de Educación Universitaria);

c) La universidad "A" tiene elevada reputación en la comunidad local e internacional.

\subsection{Instrumento de medición}

Se diseñó como instrumento de medición una guía de proposiciones de 11 ítems. Los diez primeros ítems eran proposiciones para responder de acuerdo con una escala de Likert 
que valoraba el nivel de aceptación de cada ítem en un intervalo de 1 a 5; estar totalmente de acuerdo se valoraba con 1 y estar totalmente en desacuerdo se valoraba con 5. El último ítem consistió en la siguiente pregunta ¿Qué limitación o limitaciones encuentra usted en el uso del método de casos para enseñar un curso de emprendimiento? Esta interrogante se orientaba a recopilar comentarios de los participantes sobre las limitaciones del método de casos.

Para diseñar los ítems utilizados, nos basamos en los estudios de Bloom (1956) y Anderson con Krathwohl (2001), como marco conceptual relacionado con el proceso de aprendizaje en el ámbito del conocimiento. Sobre la base de dichos estudios, se desarrollaron los diez primeros ítems y luego se procedió a su validación para asegurar su representatividad de acuerdo con el objetivo de la investigación. Para validar los ítems se recurrió a 8 expertos (Cuadro 3).

De los expertos, el 75\% son académicos, con experiencia mínima de diez años en la enseñanza mientras que el $25 \%$ son emprendedores que tienen más de quince años de experiencia creando, administrando y vendiendo pequeños negocios. En este contexto, la confiabilidad de dicho instrumento fue determinada mediante el coeficiente Alfa de Cronbach, el cual mide la fiabilidad de la consistencia interna de los ítems. Para la presente investigación se obtuvo un alfa de Cronbach de 0.80 que refleja un buen nivel de confiabilidad del instrumento aplicado. La información se ingresó manualmente y se trabajó en formato Excel.

Cuadro 3 - Expertos participantes en la validación de los ítems

\begin{tabular}{|c|c|c|c|}
\hline Identidad $(*)$ & Rol & Profesión base & Institución / empresa \\
\hline Experto 1 & Académico & $\begin{array}{c}\text { Historiadora- Escritora } \\
\text { de casos }\end{array}$ & HEC de Montreal Canadá \\
\hline Experto 2 & Académico & Contador & $\begin{array}{l}\text { Pontificia Universidad } \\
\text { Católica de Sao Paulo }\end{array}$ \\
\hline Experto 3 & Académico & $\begin{array}{l}\text { Licenciada en } \\
\text { Informática }\end{array}$ & $\begin{array}{c}\text { Universidad La Salle } \\
\text { México }\end{array}$ \\
\hline Experto 4 & Académico & Administrador & $\begin{array}{l}\text { Universidad Nacional } \\
\text { Mayor de San Marcos }\end{array}$ \\
\hline Experto 5 & Académico & $\begin{array}{l}\text { Licenciado en } \\
\text { Educación }\end{array}$ & $\begin{array}{l}\text { Pontificia Universidad } \\
\text { Católica del Perú }\end{array}$ \\
\hline Experto 6 & Académico & $\begin{array}{l}\text { Licenciada en } \\
\text { Educación }\end{array}$ & $\begin{array}{l}\text { Pontificia Universidad } \\
\text { Católica del Perú }\end{array}$ \\
\hline Experto 7 & Emprendedor & $\begin{array}{c}\text { Administrador de } \\
\text { empresas }\end{array}$ & Overflow - Lima, Perú \\
\hline Experto 8 & Emprendedor & $\begin{array}{c}\text { Bachiller en Ingeniería } \\
\text { electrónica }\end{array}$ & Digital 20 - Lima, Perú \\
\hline
\end{tabular}

(*) Por confidencialidad, se mantiene en reserva el nombre de los expertos.

Elaboración: propia 


\section{RESULTADOS Y DISCUSIÓN}

El trabajo de investigación en campo produjo interesantes hallazgos que permiten valorar el uso del método de casos como herramienta didáctica en los cursos de emprendimiento.

Ítem 1: El 91.7\% de los participantes manifiesta estar totalmente de acuerdo con que la metodología de casos es una buena herramienta de enseñanza en un curso de emprendimiento, mientras que un $8.3 \%$ estuvo parcialmente de acuerdo con ello. Esta situación indica una elevada aceptación para usar la metodología, por lo cual los docentes que enseñen la materia, pueden incorporarla como una herramienta didáctica apropiada.

Ítem 2: El $86.7 \%$ de los participantes indica estar totalmente de acuerdo con que la metodología de casos es una fuente de conexión con la realidad empresarial, mientras que un $11.7 \%$ estuvo parcialmente de acuerdo. Esto refleja la importancia de elaborar y/o trabajar con casos sobre emprendedores, en los cuales se pueda conocer aspectos cualitativos del emprendedor, su familia, sus colaboradores y la información verificable del entorno sectorial. Todo ello permitirá una mejor vinculación con el espíritu empresarial.

Ítem 3: El 48.3\% de los participantes está totalmente de acuerdo con la limitación que los estudiantes no hayan realizado la lectura previa al desarrollo del caso, esto significa que sí existe un nivel de compromiso de los estudiantes con la herramienta didáctica.

Ítem 4: El $88.3 \%$ de los participantes se mostró completamente de acuerdo con el hecho que la metodología de casos refuerza el conocimiento de temas sobre emprendimiento. Entonces, es necesario suministrar un marco referencial teórico por cada unidad del curso, pero la posibilidad de fortalecer el conocimiento se fortalece con el uso de casos

Ítem 5: El 70.0\% de los participantes concuerda completamente con la proposición que la metodología de casos ayuda a una mejor comprensión de los temas de emprendimiento, mientras que el $28.3 \%$ dijo estar parcialmente de acuerdo. La situación refleja la importancia de combinar esta herramienta con otras herramientas como algunas de las planteadas en el cuadro 3. Los participantes también demandan invitar a la clase a un emprendedor para que haga una exposición en vivo o vía la plataforma Zoom.

Ítem 6: El 76.7\% de los participantes admite estar completamente de acuerdo con que la metodología de casos posibilita aplicar la información del curso de emprendimiento a situaciones nuevas, mientras que el 21.7 está parcialmente de acuerdo. Obviamente, analizar distintos problemas que enfrentan los emprendedores en la búsqueda por crear y evaluar soluciones para la toma de decisiones, ayuda a aplicar los conocimientos que se van adquiriendo.

Ítem 7: El 75.0\% de los participantes señala estar completamente de acuerdo respecto al hecho que la metodología de casos permite analizar diferentes situaciones vinculadas a una diversidad de temas sobre la actividad emprendedora, mientras que el $20.0 \%$ está parcialmente de acuerdo. Los resultados revelan la importancia de trabajar casos emprendedores relacionados con problemas que ocurren en las distintas áreas funcionales y en diversos giros de negocio,

Ítem 8: El 58.3\% de los participantes está completamente de acuerdo con que la metodología de casos apoya el proceso de sintetizar e integrar conocimientos en los temas de emprendimiento, mientras que el $36.7 \%$ se encuentra parcialmente de acuerdo. Esta situación 
refleja la importancia de combinar los casos con otras herramientas didácticas, según la característica de los estudiantes, la habilidad del profesor y el perfil de egreso.

Ítem 9: El 66.7\% de los participantes admite estar completamente de acuerdo con que la metodología de casos contribuye a emitir juicios personales con un objetivo determinado, acerca de un tema, sobre la base de criterios preestablecidos, mientras que el $36.7 \%$ sostiene estar parcialmente de acuerdo. Ante este resultado, la inclusión de otras herramientas como el aprendizaje basado en proyectos, el trabajo colaborativo y el role playing, pueden complementar favorablemente el método de casos.

Ítem 10: El 76.7\% de los participantes está completamente de acuerdo con la proposición que en el futuro se usará, en mayor medida, el método de casos para optimizar el proceso de aprendizaje en un curso de emprendimiento. Esta situación predice la trascendencia que el método de casos tendrá como herramienta didáctica en los cursos de emprendimiento que se impartirán en el largo plazo, por lo cual los profesores requieren prepararse para utilizarlo, resultando también valiosa la disponibilidad de una base de datos de casos.

Ítem 11: El 75.0\% de los participantes está de acuerdo con que el método de casos no presenta limitaciones en la enseñanza de un curso de emprendimiento porque permite conectarse con el mundo real de los pequeños negocios y ayuda a conocer la problemática que enfrentan los emprendedores cuando administran sus negocios. Asimismo, dijeron que la adecuada elaboración del caso, el uso de información cuantitativa actualizada, las preguntas motivadoras para discutir en clase y la experiencia del profesor en el manejo de la metodología, contribuyen a lograr mejores aprendizajes.

Por otro lado, acerca de las limitaciones que presenta el método del caso los participantes señalaron: que es necesario analizar casos de diferentes países y culturas para comprender de manera integral la actividad emprendedora; que el estudiante no tenga experiencia previa para analizar todo el panorama del caso planteado; que no se proporcionen todos los datos necesarios para analizar el caso; que el número de horas del curso necesita dosificarse; y que no es posible conocer presencialmente a los emprendedores mostrados en los casos dado que les gustaría oír de primera mano su opinión e historia.

\section{CONSIDERACIONES FINALES}

El emprendimiento es una actividad vinculada a la creación de nuevos negocios y la cultura para hacer empresa, lo cual demanda adquirir un conjunto de actitudes, habilidades y conocimientos para impulsar la actividad emprendedora en cualquier campo del quehacer humano, con el fin de crear sostenibilidad y crecimiento económico.

La educación emprendedora se ha convertido en un aspecto esencial para los gobiernos que buscan promover el empleo, hacer más equitativa la distribución del ingreso, crear un atractivo entorno para crear nuevos negocios $\mathrm{y}$, en general, promover mejores condiciones de vida para sus ciudadanos.

Las herramientas didácticas son mecanismos para la construcción de aprendizajes, por ello, la elección de determinadas herramientas depende de las características de los estudiantes, la naturaleza del curso, el nivel de estudios cursado, el perfil de egreso, la experiencia del profesor y los recursos disponibles. 
El método del caso es una herramienta didáctica valorada por los estudiantes en la enseñanza de un curso de emprendimiento porque ayuda a conectar al emprendedor con el estudiante y trasmite las vivencias y problemas que enfrentan los emprendedores cuando manejan pequeños negocios, lo cual promueve aprendizajes significativos.

Es necesario efectuar nuevas investigaciones para profundizar el nivel de conocimiento existente sobre el uso de del uso del método del caso como herramienta didáctica en un curso de emprendimiento, ampliando el tamaño de la muestra e incorporando otras variables que posibiliten obtener resultados con mayor alcance.

\section{REFERENCIAS}

Anderson, L. W. and Krathwohl, D.R., et al. (2001). A taxonomy for learning, teaching and assessing: A revision of Bloom's taxonomy of educational objectives. New York: Longmans

Boehrer J. and M. Linsky. "Teaching with cases: learning to question". New directions for teaching and learning No. 42. San Francisco: Jossey-Bass, 1990. Recuperado el 8 de junio de 2014 de http://www.soc.ucsb.edu/projects/casemethod/casediscussion.pdf

Bloom, B.S. and Krathwohl, D. R. (1956). Taxonomy of Educational Objectives: The Classification of Educational Goals, by a committee of college and university examiners. Handbook I: Cognitive Domain. NY, NY: Longmans, Green and Co. Ltd.

Boldureanu, G. et al. (2020). Entrepreneurship education through successful entrepreneurial models in higher education systems. Sustainability, 12, 1267.

Brenes E. y Haar J. (2017). El futuro del emprendimiento en Latinoamérica. Segunda edición. México D.F.: Cengage Learning Editores.

Camacho, M. (2011). Historias empresariales en los estudios de caso. Pensamiento y Gestión, № 31. Universidad del Norte, p. 196-210.

CEPAL (2013). La Unión Europea y América Latina y el Caribe: Inversiones para el crecimiento, la inclusión social y la sostenibilidad ambiental. Santiago: CEPAL.

Comisión Europea (2016). EntreComp: TheEntrepreneurship Competence Framework. Recuperado el 07 de junio de 2021 de

http://publications.jrc.ec.europa.eu/repository/bitstream/JRC101581/lfna27939enn.pdf

Churches, A. (2009). Taxonomía de Bloom para la era digital. Recuperado el 10 de junio de http://edorigami.wikispaces.com

Dirección de Investigación y Desarrollo Educativo, Instituto Tecnológico de Monterrey (s.f.). Las estrategias y técnicas didácticas en el rediseño: Capacitación en estrategias y técnicas didácticas. Recuperado el 22 de junio de 2021 de http://sitios.itesm.mx/va/dide/documentos/inf-doc/Est_y_tec.PDF

Dirección General de Política de la Pyme (2010). El fomento de la iniciativa emprendedora en el sistema educativo en España. Madrid: Ministerio de Industria, Turismo y Comercio. Recuperado el 15 de junio de 2021 de

http://www.ipyme.org/Publicaciones/FomentoIniciativaEmprendedora.pdf

Drucker, P. (1985). The entrepreneurial mystique. Inc. 7(10), p. 34-58.

Refas - ISSN 2359-182X v.7, n.6

Agosto de 2021 
Estrada, A. y Alfaro, K. Mendives (2013). Investigación Bibliotecológica, Vol. 29, Núm. 65, enero/abril, 2015, México, p. 195-212.

Filion, L.J.; Martínez, L. y Mejía-Morelos, J. (2011). Administración de Pymes: emprender, dirigir y desarrollar empresas. D.F.: Pearson Educación

Filion, L.J. (1999). Empreendedorismo: Empreendedores e proprietários-gerentes de pequenos negócios. Revista de Administração, São Paulo, Vol. 34, n. 2, p. 5-28.

Hardie, B.; Highfield, C. and Lee, K. (2020). Entrepreneurship education today for students' unknown futures. Journal of Pedagogical Research, 4(3), p. 401-417

Garvin DA. Making the case. Harv Mag 2003; 106(1), p. 56-65.

Gutiérrez-Ponce, H. et al. (2020). El método del caso en la formación de economistas: elaboración y aplicación. magis, Revista Internacional de Investigación en Educación, 12 (25), 145-168.

Harvard Law School (s/f) Recuperado el 19 de julio de 2021 de https://casestudies.law.harvard.edu/the-case-study-teaching-method/

INACAP. (2017). Subdirección de Currículum y Evaluación, Dirección de Desarrollo Académico, Vicerrectoría Académica de Pregrado, Universidad Tecnológica de Chile Manual de Estrategias Didácticas: Orientaciones para su selección. Santiago: Ediciones INACAP.

Isaacs, G. (1996). Bloom's taxonomy of educational objectives. Queensland: Teaching and Educational Development Institute.

Krathwohl, D.R. (2002). A Revision of Bloom's Taxonomy: An Overview. THEORY INTO PRACTICE, Volume 41, Number 4, College of Education, The Ohio State University, 212218.

Neck, H. M. and Greene, P. G. (2011). Entrepreneurship Education: Known Worlds and New Frontiers. Journal of Small Business Management, 49(1), 55-70.

Neck, H. M.; Neck, Ch. and Murray, E. (2020). Entrepreneurship: the practice and mindset. Second Edition. Thousand Oaks: SAGE Publishing.

Nueno, P. (2009). Emprendiendo hacia el 2020: una renovada perspectiva global del arte de crear empresas y sus artistas. Barcelona: Ediciones Deusto.

Ogliastri, E. (2009). El método de casos. Cali, Colombia: Universidad ICESI CREA. Serie Cartillas Docentes.

Paños, J. (2017). Educación emprendedora y metodologías activas para su fomento. Revista Electrónica Interuniversitaria de Formación del Profesorado, 20 (3), 33-48.

Real Academia de la Lengua Española, RAE. Recuperado el 22 de junio de 2021 de http://www.rae.es

Reynolds, P. et al. (2005). Global Entrepreneurship Monitor: Data collection design and implementation 1998-2003. Small Business Economics, 24(3), 205-231.

Rosker, E. J. (2006), "El método de casos como herramienta transformadora de la sociedad", en Universidad y Empresa, núm. 11, pp. 109-122.

Serida, J. et al. (2018); Global Entrepreneurship Monitor Perú 2017-2018. Lima: Universidad ESAN. 
Shane, S. and Venkataraman, S. (2000). The promise of entrepreneurship as a field of research. Academy of Management Review, Vol. 25, n. 1, pp. 217-226.

The Quality Assurance Agency for Higher Education, QAA. (2018). Enterprise and Entrepreneurship Education: Guidance for UK Higher Education Providers. Recuperado el 10 de junio de 2021 de

https://www.qaa.ac.uk/docs/qaas/enhancement-and-development/enterprise-andentrpreneurship-education-2018.pdf?sfvrsn=15f1f981_8

Serviço Brasileiro de Apoio às Micro e Pequenas Empresas (SEBRAE). Recuperado el 13 de julio de 2021 de https://atendimento.sebrae-sc.com.br/blog/o-que-e-empreendedorismo/

Schumpeter, J.A. (1912). The Theory of Economic Development. Oxford University Press, Oxford.

Wassermann, S. (1994), El estudio de casos como método de enseñanza. Buenos Aires: Amorrortu Editores.

Wennekers, A.R.M. and Thurik A.R. (1999). "Linking Entrepreneurship and Economic Growth", Small Business Economics, Vol. 13, pp. 27-55

Zapata-Ros, M. Teorías y modelos sobre el aprendizaje en entornos conectados y ubicuos. Bases para un nuevo modelo teórico a partir de una visión crítica del "conectivismo". Education in the knowledge society, Vol. 16, Nro. 1, 2015, pp. 69-102. 